\title{
Dopamine Surface Modification of Trititanate Nanotubes: Proposed In-Situ Structure Models
}

\author{
Ruochen Liu, Xuejian Fu, Congyi Wang, and Graham Dawson*[a]
}

Abstract: Two models for self-assembled dopamine on the surface of trititanate nanotubes are proposed: individual monomer units linked by $\pi-\pi$ stacking of the aromatic re- gions and mono-attached units interacting through hydrogen bonds. This was investigated with solid state NMR spectroscopy studies and powder X-ray diffraction.

\section{Introduction}

Surface functionalisation is a facile tool for modifying the properties of nanomaterials. Since first reported in 2007, polydopamine coatings have gained much research interest in a variety of fields and applications, including biocompatible surface modifications, ${ }^{[1]}$ energy devices ${ }^{[2]}$ and nanomaterial functionalisation. ${ }^{[3-5]}$

In recent years inorganic nanostructured materials have been extensively investigated. ${ }^{[6,7]}$ Amongst this class of compounds $\mathrm{TiO}_{2}$ has received much attention owing to its photocatalytic properties, including high oxidative power and longterm stability, ${ }^{[8,9]}$ which have led to its use in applications such as self-cleaning surface coatings. In 1998, Kasuga et al. reported the preparation of titania nanotubes ${ }^{[10]}$ by the hydrothermal treatment of $\mathrm{TiO}_{2}$ with $10 \mathrm{~m} \mathrm{NaOH}$. These nanotubes had a porous structure and high surface area. The composition of the nanotubes was subsequently identified as trititanate, ${ }^{[11-14]}$ $\mathrm{H}_{2} \mathrm{Ti}_{3} \mathrm{O}_{7}$. We recently functionalised the surface of trititanate nanotubes with dopamine, imparting recyclable photocatalytic properties on the nanotubes. ${ }^{[15]}$ Dopamine promoted electron injection and charge separation, adding activity to the system which was lacking with the unmodified nanotubes.

The exact structure of polydopamine is still under debate. Until recently it was assumed that the structure was based on an open-chain polycatechol model, or a polymeric skeleton based on 5,6-dihydroxyindole ( $\mathrm{DHI})$, the cyclisation product of dopamine. Neither of these models are based on solid experimental evidence. In 2013 it was reported that polydopamine (pda) can be viewed as a mixture of oxidative polymerisation and physical self-assembly. ${ }^{[16,17]}$ This results in a complex mixture of cyclised and uncyclised polymer conjugates, and physical trimer units.

\footnotetext{
[a] R. Liu, X. Fu, C. Wang, Dr. G. Dawson

Department of Chemistry, Xian Jiaotong Liverpool University

111 Renai Road, Suzhou, 215123 (P.R. China)

E-mail: graham.dawson@xjtlu.edu.cn

$\square$ Supporting information for this article is available on the WWW under http://dx.doi.org/10.1002/chem.201600075.
}

In these studies investigation of the pda structure was carried out solely on pda, removed from any modified surface. In this study we have investigated the structure of dopamine modified trititante nanotubes in situ by solid state NMR spectroscopy and powder X-ray diffraction, and we propose possible modes for self-assembled linkages based on the evidence.

\section{Results and Discussion}

We have previously proposed that dopamine attached to TiNT via the enediol $-\mathrm{OH}$ groups, ${ }^{[15]}$ giving increased absorbance in the visible range of the spectrum, however the exact nature and interaction of dopamine on the surface was not further investigated. As shown in Figure 1, dopamine itself exhibits no signal between 300 and $800 \mathrm{~nm}$ in the UV/Vis. Synthesised pda exhibits obvious absorbance in this range, as do the modified nanotubes (TiNT-dopamine). The TiNT-dopamine shows absorbance across the full range and an obvious shoulder from $400 \mathrm{~nm}$. This has been attributed to excitation into a localised state. ${ }^{[5]}$ There is an obvious colour change after functionalisation, as can be seen in Figure S1 in the Supporting Information. The synthesised polydopamine colour is also shown for comparison. This led us to believe that there may be some polymerisation occurring in the dopamine before it is attached onto the TiNT surface. This was investigated further using solid state NMR spectroscopy and powder X-ray diffraction.

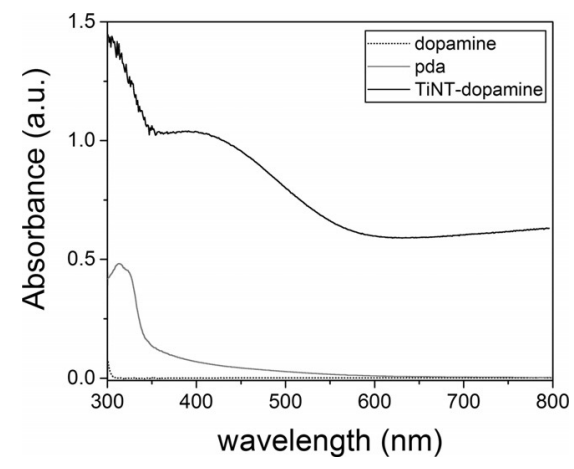

Figure 1. UV/Vis spectra of dopamine, pda and TiNT-dopamine. 
The ${ }^{13} \mathrm{C}$ solid state NMR spectra of dopamine and TiNTdopamine were measured. The peaks in the spectrum of dopamine, shown in Figure $2 \mathrm{a}$, are assigned as follows: the two carbon atoms from the chain $(\delta=32,42 \mathrm{ppm})$, and the six carbon atoms from the aromatic ring $(\delta=115,116,122,131$, $142,145 \mathrm{ppm})$. The two peaks at $\delta=142$ and $145 \mathrm{ppm}$ were assigned to the carbons attached to the $\mathrm{OH}$ groups. ${ }^{[16,17]}$ Figure $2 \mathrm{~b}$ shows the solid state NMR spectrum of TiNT-dopamine. From the spectrum, it can be seen that the peaks at about $\delta=$ 30 and $40 \mathrm{ppm}$ are similar to that of NMR spectrum of dopamine. That means the chain of dopamine is still present, and that no or limited cyclisation has occurred.

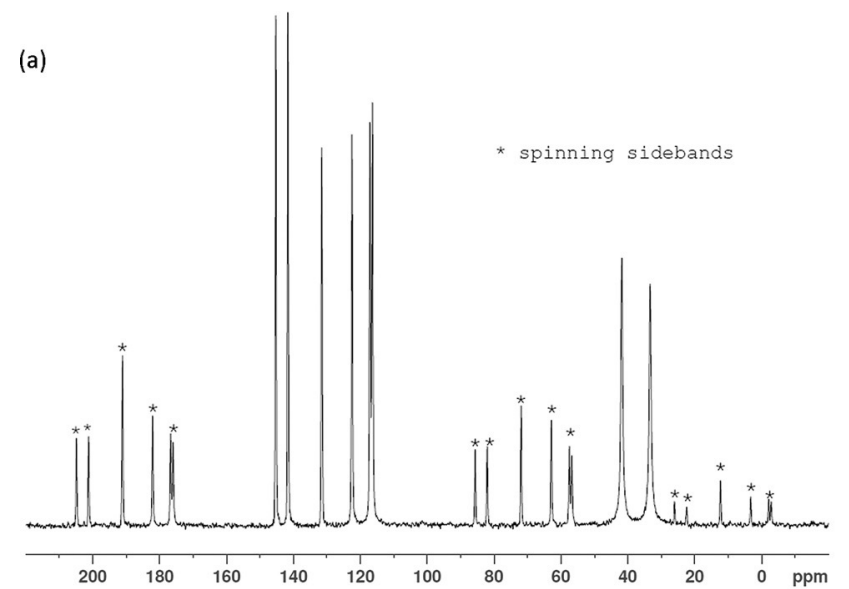

(b)

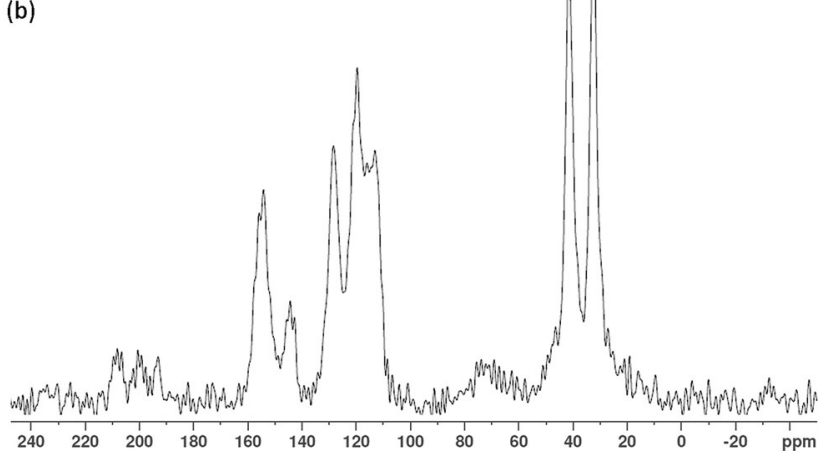

Figure $2 .{ }^{13} \mathrm{C}$ solid state NMR spectra of: a) dopamine, and b) TiNT-dopamine.

A peak shift was observed from $\delta=145$ to $155 \mathrm{ppm}$, which was caused by the replacement of $\mathrm{C}-\mathrm{OH}$ bond by the $\mathrm{C}-\mathrm{O}-\mathrm{Ti}$ bond. This suggests that the $\mathrm{C}-\mathrm{OH}$ bonds broke and $\mathrm{C}-\mathrm{O}-\mathrm{Ti}$ bonds were formed during the preparation of TiNT-dopamine, resulting in dopamine attachment through the hydroxyl group to the TiNT surface. Moreover, the continued existence of the peak at $\delta=145 \mathrm{ppm}$ suggests that mono-attached molecules may also exist in the sample. These data support the FTIR data (Figure S2 in the Supporting Information) showing the disappearance of the $-\mathrm{OH}$ bending mode after functionalisation, which is of too low a resolution to be conclusive in itself.

The peak at $\delta=131 \mathrm{ppm}$ in the spectrum of dopamine has been shifted to $\delta=128 \mathrm{ppm}$ upon surface functionalisation.
This peak is of great interest. Whilst a peak in this region is seen in the previous study by Della Vecchia et al.., ${ }^{[16]}$ it is not specifically assigned. We are proposing two possible structure models which can explain the appearance of the NMR spectrum. The first model, show in Figure $3 \mathrm{a}$, has mono-attached units interacting by hydrogen bonds. This binding mode explains the continued existence of the $\mathrm{C}-\mathrm{OH}$ peak in the spectrum. The second model, in Figure $3 \mathrm{~b}$, contains fully attached monomer units interacting through a $\pi-\pi$ stacking interaction of dopamine units on the TiNT surface. The lack of cyclisation would tend to indicate that limited polymerisation has occurred. The shift from $\delta=131$ to $128 \mathrm{ppm}$ can be designated as $\pi-\pi$ stacking between the aromatic units, causing deshielding and a downfield shift in the signal. ${ }^{[18]}$ NMR spectroscopy studies have also shown $\pi-\pi$ stacking ${ }^{[19-24]}$

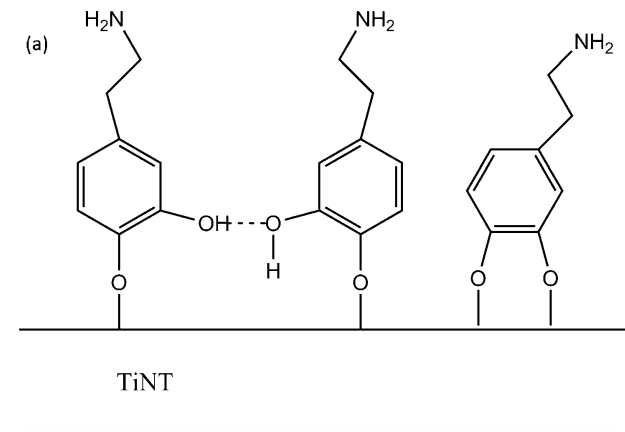

(b)

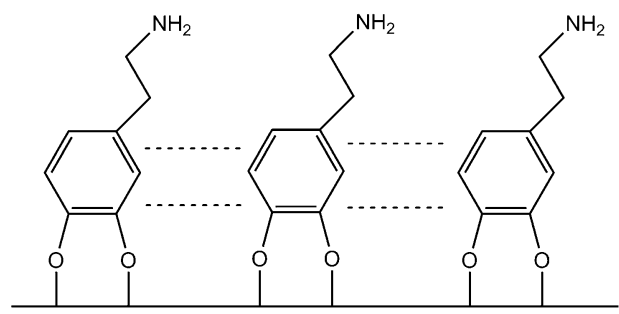

TiNT

Figure 3. Proposed structure of dopamine on TiNT surface.

In a similar surface modification situation, $\pi-\pi$ stacking has been shown to occur on tetracene on aluminium oxide substrate, resulting in a densely packed monolayer of tetracene covalently attached through $\mathrm{C}-\mathrm{O}-\mathrm{Al}$ bonds, and an almost upright arrangement of tetracene molecules. ${ }^{[25]}$ This was investigated by synchrotron X-ray reflectivity measurements.

In order to further investigate the surface arrangement of dopamine, powder X-ray diffraction (XRD) was used. The $\pi-\pi$ stacking has been shown in small and large molecules with the stacking ${ }^{[26]}$ range from 3-4 $\AA$. XRD studies of melanins and polydopamine have shown a characteristic broad peak in this range, which has been attributed to the stacking between aggregated layers. ${ }^{[27-29]}$ The XRD pattern of TiNT and TiNT-dopamine are shown in Figure 4. The peaks for TiNT are indexed ${ }^{[30]}$ 
as $\mathrm{H}_{2} \mathrm{Ti}_{3} \mathrm{O}_{7}$. Upon functionalisation, a broad peak centred at 28 degrees is observed. This corresponds to a $d$ spacing of $3.18 \AA$. This is slightly smaller than that observed in pda studies, ${ }^{[28]}$ however, within the range expected for $\pi-\pi$ stacking. ${ }^{[26]}$ We propose that the surface healing gained from functionalisation with dopamine ${ }^{[31]}$ forces the dopamine monomer units to be in closer $\pi-\pi$ interaction than would be observed in a free polymer. Along with the ${ }^{13} \mathrm{C}$ NMR spectroscopy data, this would tend to suggest that the dopamine molecules exist as discreet monomers on the TiNT surface. There is also a shift from 25.22 to 24.2 degrees observed for the (110) peak of $\mathrm{H}_{2} \mathrm{Ti}_{3} \mathrm{O}_{7}$ of the TiNT, which would correspond to a slightly larger $d$ spacing. However, the other peaks in the XRD pattern are not altered (Figure $\mathrm{S} 3$ in the Supporting Information).

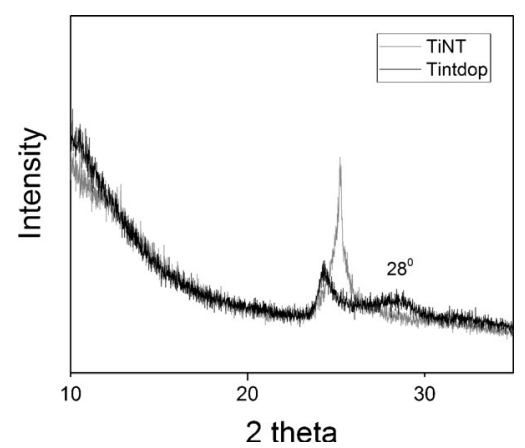

Figure 4. Powder X-ray diffraction pattern of TiNT and TiNT-dopamine.

Pda has previously shown a complex mixed structure, however our data suggest that on TiNT surface, the monomer units are arranged as discreet units in close contact with each other.

Transmission electron microscopy (TEM) images in Figure 5 shows TiNT and TiNt-dopamine after modification. As can be seen, the TiNT after dopamine modification have a thin amorphous coating of 1-2 $\mathrm{nm}$. Through $\mathrm{CHN}$ elemental analysis and thermogravametric analysis (Figure $\mathrm{S} 4$ in the Supporting Information) the amount of dopamine in the TiNT-dopamine sample was $4.3 \mathrm{wt} \%$. Assuming a coverage $\mathrm{e}^{[5]}$ of $19.6 \AA^{2}$, then this corresponds to a $13 \%$ surface coverage (BET surface area of $249.4 \mathrm{~m}^{2} \mathrm{~g}^{-1}$ ). The internal surface area of the nanotube will not be available for functionalisation, resulting in the observed external surface coating. The existence of a dopamine coating on the TiNT is also confirmed by scanning electron microscopy and elemental analysis mapping (Figures S5 and S6 in the Supporting Information), where the presence of carbon is clearly observed, and is absent from the pristine TiNT.

\section{Conclusions}

In this study we have proposed two modes for self-assembled dopamine on the surface of trititanate nanotubes: individual monomer units linked by $\pi-\pi$ stacking of the aromatic regions and mono-attached units interacting through hydrogen bonds. This result indicates that pda coatings may involve more monomer physical assembly than previously thought, and
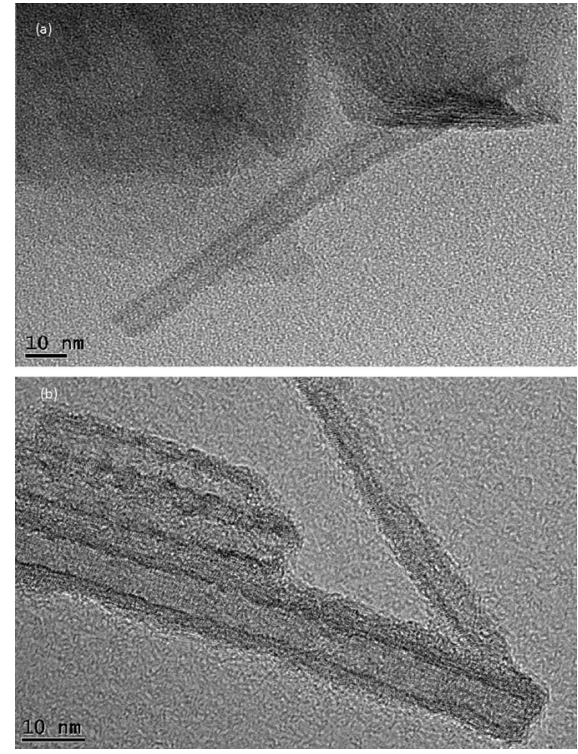

Figure 5. TEM images of: a) TiNT, and b) TiNT-dopamine showing thin dopamine coating.

offers an insight into functional use of pda. This thin dopamine coating may have applications in multifunctional biocompatible systems.

\section{Experimental Section}

Synthesis of dopamine modified trititanate nanotubes (TiNT-dopamine) was carried out following the literature procedure, ${ }^{[11]}$ specifically anatase $\mathrm{TiO}_{2}(1 \mathrm{~g})$ was added to $10 \mathrm{M} \mathrm{NaOH}$ solution $(20 \mathrm{~mL})$. The resultant slurry was transferred to a TEFLON vessel and sealed in an autoclave at $140^{\circ} \mathrm{C}$ for $72 \mathrm{~h}$. The white solid was removed and washed with water, $0.1 \mathrm{M} \mathrm{HCl}$ solution, and finally by copious amounts of distilled water until the $\mathrm{pH}$ value of the effluent was neutral. The surface-modified nanotubes were prepared by mixing the solid with $10 \mathrm{wt} \%$ of dopamine hydrochloride. The suspension was heated at $70^{\circ} \mathrm{C}$, overnight, then washed with water and ethanol to remove any excess ligand.

\section{Synthesis of pda}

Dopamine was allowed to oxidise in $\mathrm{NaOH}$ solution until it became black. Water was removed, leaving a brown/black viscous substance.

\section{Solid state NMR spectroscopy}

${ }^{13} \mathrm{C}$ NMR spectra were measured on a Bruker Advance III 400 spectrometer equipped with a $4 \mathrm{~mm} \mathrm{H/X}$ DVT probe. The spinning speeds were set at $8 \mathrm{kHz}$. Cross polarisation experiments were with a contact time of $1000 \mu \mathrm{s}$. The chemical shifts of the spectra were referenced with respect to TMS $(0 \mathrm{ppm})$ by using admantane as second standard (38.484 ppm). For TiNT-dopamine 20480 scans were recorded. For pure dopamine 160 scans were recorded. UV/ Vis spectra were measured as powders on an Agilent Cary 300 spectrometer fitted with a diffuse reflectance accessory. All Fourier transform infrared spectroscopy (FTIR) samples were measured on an Agilent Cary 660 spectrometer. All of the samples were recorded in a $\mathrm{KBr}$ matrix. Transmission electron microscopy was carried 
out on an FEl Tecnai F20 operating at 200 kV. Scanning electron microscope images and elemental analysis were carried out on an Agilent 8500 FE-SEM (operating at $1 \mathrm{kV}$ ) and a JEOL JSM-6510 equipped with a Penta FET Oxford EDX detector operating at $25 \mathrm{kV}$, respectively. Powder $\mathrm{X}$-ray diffraction was recorded on a Bruker D8 advance diffractometer with $\mathrm{Cu}_{\mathrm{k \alpha}}$ at $40 \mathrm{kV}$ and $40 \mathrm{~mA}$.

\section{Acknowledgements}

This work was supported by Xian Jiaotong Liverpool University Research Development Fund and SURF projects.

Keywords: nanomaterials · polymers · self-assembly $\cdot$ surface analysis

[1] J. Sedó, J. Saiz-Poseu, F. Busque, D. Ruiz-Molina, Adv. Mater. 2013, 25, 653.

[2] M. H. Ryou, Y. M. Lee, J. K. Park, J. W. Choi, Adv. Mater. 2011, 19, 3066.

[3] A. Postma, Y. Yan, Y. Wang, A. N. Zelikin, E. Tjipto, F. Caruso, Chem Mater. 2009, 21, 3042.

[4] L. Zhu, Y. Lu, Y. Wang, L. Zhang, W. Wang, Appl. Surf. Sci. 2012, 258, 5387.

[5] T. Rajh, L. X. Chen, K. Lucas, T. Liu, M. C. Thurnauer, D. M. Tiede, J. Phys. Chem. B 2002, 106, 10543

[6] R. Tenne, L. Margulis, M. Genut, G. Hodes, Nature 1992, 360, 444.

[7] Y. Feldman, E. Wasserman, D. J. Srolovitz, R. Tenne, Science 1995, 267, 222

[8] P. Hoyer, Langmuir 1996, 12, 1411.

[9] A. Fujishima, K. Hashimoto, T. Watanabe, TiO2 Photocatalysis: Fundamentals and Applications, BKC, Tokyo, 1999.

[10] T. Kasuga, M. Hiramatsu, A. Hoson, T. Sekino, K. Niihara, Langmuir 1998 , $14,3160$.

[11] A. Thorne, A. Kruth, D. Tunstall, J. T. S. Irvine, W. Zhou, J. Phys. Chem. A 2005, 109, 5439 .

[12] Q. Chen, W. Zhou, G. Du, L.-M. Peng, Adv. Mater. 2002, 14, 1208.
[13] S. Zhang, L.-M. Peng, Q. Chen, G. H. Du, G. Dawson, W. Zhou, Phys. Rev. Lett. 2003, 91, 256103

[14] D. V. Bavykin, J. M. Friedrich, F. C. Walsh, Adv. Mater. 2006, 18, 2807.

[15] G. Dawson, J. Liu, L. Lu, W. Chen, ChemCatChem 2012, 4, 1133.

[16] N. F. Della Vecchia, R. Avolio, M. Alfe, M. E. Errico, A. Napolitano, M. d'Ischia, Adv. Funct. Mater. 2013, 23, 1331.

[17] S. Hong, Y. S. Na, S. Choi, I. T. Song, W. Y. Kim, H. Lee, Adv. Funct. Mater. 2012, 22, 4711

[18] R. S. Macomber, A Complete Introduction to Modern Spectroscopy, Wiley, 1997, chapter 6.

[19] C. Moon, G. Brunklaus, D. Sebastiani, Y. Rudzevich, V. Bohmer, H. W. Spiess, Phys. Chem. Chem. Phys. 2009, 11, 9241.

[20] S. Standara, K. Bouzkova, M. Straka, Z. Zacharova, M. Hocek, J. Marek, R. Marek, Phys. Chem. Chem. Phys. 2011, 13, 15854.

[21] M. Balog, I. Grosu, G. Ple, Y. Ramondenc, E. Condamine, R. A. Varga, J. Org. Chem. 2004, 69, 1337.

[22] T. Nakano, Polymer J. 2010, 42, 103-123.

[23] F. R. Fischer, W. B. Schweizer, F. Diederich, Chem. Commun. 2008, 4031

[24] F. Cozzi, M. Cinquini, R. Annuniziata, T. Dwyer, J. S. Siegel, J. Am. Chem. Soc. 1992, 114, 5729.

[25] G. S. Tulevski, Q. Miao, M. Fukoto, R. Abram, B. Ocko, R. Pindak, M. L. Steigerwald, C. R. Kagan, C. Nuckolls, J. Am. Chem. Soc. 2004, 126, 15048.

[26] A. A. R. Watt, J. P. Bothma, P. Meredith, Soft Matter 2009, 5, 3754.

[27] V. Capozzi, G. Perna, P. Carmone, A. Gallone, M. Lastella, E. Mezzenga, G. Quartucci, M. Ambrico, V. Augelli, P. F. Biagi, T. Ligonzo, A. Minafra, L. Schiavulli, M. Pallara, R, Cicero, Thin Solid Films 2006, 511-512, 362

[28] H. Fan, X. Yu, Y. Liu, Z. Shi, H. Liu, Z. Nie, D. Wu, Z. Jin, Soft Matter 2015, $11,4621$.

[29] M. d'Ischia, A. Napolitano, A. Pezzella, P. Meredith, Angew. Chem. Int. Ed. 2009, 48, 3914; Angew. Chem. 2009, 121, 3972.

[30] G. Dawson, W. Chen, T. Zhang, Z. Chen, X. Cheng, Solid State Sci. 2010, $12,2170$.

[31] Z. V. Saponjic, N. M. Dimitrijevic, D. M. Tiede, A. J. Goshe, X. Zuo, L. X. Chen, A. S. Barnard, P. Zapol, L. Curtiss, T. Rajh, Adv. Mater. 2005, 17, 965.

Received: January 8, 2016

Published online on March 22, 2016 